\title{
A Simple Greedy Algorithm for Dynamic Graph Orientation*
}

\author{
Edvin Berglin ${ }^{1}$ and Gerth Stølting Brodal ${ }^{2}$ \\ 1 Department of Computer Science, Aarhus University, Aarhus, Denmark \\ berglin@cs.au.dk \\ 2 Department of Computer Science, Aarhus University, Aarhus, Denmark \\ gerth@cs.au.dk
}

\begin{abstract}
Graph orientations with low out-degree are one of several ways to efficiently store sparse graphs. If the graphs allow for insertion and deletion of edges, one may have to flip the orientation of some edges to prevent blowing up the maximum out-degree. We use arboricity as our sparsity measure. With an immensely simple greedy algorithm, we get parametrized trade-off bounds between outdegree and worst case number of flips, which previously only existed for amortized number of flips. We match the previous best worst-case algorithm (in $\mathcal{O}(\log n)$ flips) for general arboricity and beat it for either constant or super-logarithmic arboricity. We also match a previous best amortized result for at least logarithmic arboricity, and give the first results with worst-case $\mathcal{O}(1)$ and $\mathcal{O}(\sqrt{\log n})$ flips nearly matching degree bounds to their respective amortized solutions.
\end{abstract}

1998 ACM Subject Classification G.2.2 Graph Theory

Keywords and phrases Dynamic graph algorithms, graph arboricity, edge orientations

Digital Object Identifier 10.4230/LIPIcs.ISAAC.2017.12

\section{Introduction}

An important building block in algorithmic theory and practice is the ability to store graphs with low memory usage and fast query times. Classical storage methods are edge lists and adjacency matrix, but both have pitfalls for sparse graphs: adjacency matrices use too much memory, while edge lists can have slow adjacency queries and/or updates on high-degree vertices. Much research has been devoted to improving these simple methods. The graph parameter arboricity $\alpha$ is a well-known measure of a graph's sparsity, which captures the minimum number of forests the edges of a graph can be partitioned into. Kannan et al. [6] showed how to efficiently store static graphs with low arboricity and supporting fast $(\mathcal{O}(\alpha)$ time) adjacency queries in the worst case.

Brodal and Fagerberg [3] extended this idea to consider dynamic graphs, where edges may be arbitrarily inserted or deleted. If the arboricity of the graphs remains bounded by a constant $\alpha$, the forest partitions may be forced to change due to the updates. The authors deal with this by considering the problem of orienting the edges of the dynamic graph as in [6], but by re-orienting ("flipping") edges as needed to maintain low out-degree. They gave a simple greedy algorithm and proved that its amortized number of flips was $\mathcal{O}(1)$-competitive to the number of flips made by any other algorithm - even if that other algorithm is afforded unlimited computational resources and knowledge of the entire sequence

\footnotetext{
* Work supported by the Danish National Research Foundation grant DNRF84 through the Center for Massive Data Algorithmics (MADALGO).
} 
Table 1 Previous and new results for the dynamic edge orientation of dynamic graphs with bounded arboricity $\alpha$. Flip bounds are either amortized (am.) or worst-case (w.c.) per update.

\begin{tabular}{lcccl} 
Reference & Out-degree & Flips & $\alpha$ known & Note \\
\hline Brodal \& Fagerberg [3] & $\mathcal{O}(\alpha)$ & $\mathcal{O}(\log n)$ am. & yes & $\Omega(n)$ worst-case flips \\
Kowalik [8] & $\mathcal{O}(\alpha \log n)$ & $\mathcal{O}(1)$ am. & yes & uses alg. from [3] \\
Kopelowitz et al. [7] & $\mathcal{O}(\alpha+\log n)$ & $\mathcal{O}(\alpha+\log n)$ w.c. & no & \\
Kopelowitz et al. [7] & $\mathcal{O}\left(\frac{\log n}{\log \log n}\right)$ & $\mathcal{O}\left(\frac{\log n}{\log \log n}\right)$ w.c. & no & if $\alpha=\mathcal{O}(\sqrt{\log n})$ \\
He et al. [5] & $\mathcal{O}(\alpha \sqrt{\log n})$ & $\mathcal{O}(\sqrt{\log n})$ am. & yes & uses alg. from [3] \\
He et al. [5] & $\mathcal{O}(\alpha \log n)$ & $\mathcal{O}(\alpha \log n)$ w.c. & no & \\
\hline New (Corollary 18) & $\mathcal{O}(\alpha+\log n)$ & $\mathcal{O}(\log n)$ w.c. & no & \\
New (Corollary 19) & $\mathcal{O}(\alpha \log n)$ & $\mathcal{O}(\sqrt{\log n})$ w.c. & no & \\
New (Corollary 20) & $\mathcal{O}(\log n)$ & $\mathcal{O}(\alpha \sqrt{\log n})$ w.c. & yes & if $\alpha=\mathcal{O}(\sqrt{\log n})$ \\
New (Corollary 17) & $\mathcal{O}\left(\alpha \log ^{2} n\right)$ & $\mathcal{O}(1)$ w.c. & no & \\
New (Corollary 16) & $\mathcal{O}\left(\frac{\alpha \log ^{2} n}{f(n)}\right)$ & $\mathcal{O}(f(n))$ w.c. & no & if $f(n)=\mathcal{O}(\log n)$ \\
\hline
\end{tabular}

of updates in advance. In this paper, we will use the term 'offline strategy' to describe such an algorithm. In particular, Brodal and Fagerberg showed how to maintain the out-degrees bounded by $\mathcal{O}(\alpha)$ with $\mathcal{O}(\log n)$ amortized flips, where $n$ is the number of vertices in the graph. They also gave a lower bound of $\Omega(n)$ flips for maintaining the out-degrees bounded by $\alpha$. It is not hard to see that this bound holds even for $\alpha=1$.

Kowalik [8] gave another offline strategy and applied it to the algorithm by Brodal and Fagerberg, getting $\mathcal{O}(\alpha \log n)$ out-degree in constant amortized flips, demonstrating that a reasonable trade-off was possible. Both the algorithms of Brodal and Fagerberg [3] and Kowalik [8] need to know, and use as a parameter, a bound on the arboricity of the graph.

Kopelowitz et al. [7] later found a different algorithm, which came with slightly worse bounds but in the worst case rather than amortized. Their algorithm maintains $\mathcal{O}(\alpha+\log n)$ out-degree with $\mathcal{O}(\alpha+\log n)$ flips, without knowing $\alpha$. However, if $\alpha$ is known, they give an alternate algorithm with somewhat faster running time but otherwise equal bounds. Also, if $\alpha=\mathcal{O}(\sqrt{\log n})$, both bounds can be improved slightly to $\mathcal{O}(\log n / \log \log n)$ due to some freedom in setting the base of the logarithmic terms.

He et al. [5] gave a new offline strategy with a parametrized trade-off between out-degree and flips, generalizing the two strategies in [3] and [8]. When applied to the algorithm by Brodal and Fagerberg it achieves $\mathcal{O}(\alpha \sqrt{\log n})$ out-degree with $\mathcal{O}(\sqrt{\log n})$ amortized flips. They also give another algorithm with worst-case bounds, nearly matching those in [7] but with somewhat simpler pseudocode.

The problem was originally motivated by quick adjacency queries [6]. But rather than making an explicit dictionary data structure, we focus on the problem of dynamically flipping edges to guarantee low maximum out-degree. This allows us to ignore lower bounds for dictionary operations, and we deliberately omit comparisons of update time complexity as they might be skewed unfairly in our favor. It is straightforward to create such a data structure on top of our machinery, should one so desire, by extending our solution to report which edges are flipped. This allows programmers to tailor the balance between update and query time to suit their own needs.

Dynamic edge orientations have recently become a very popular building block in dynamic graph algorithms, especially for maintaining maximal matchings; see e.g. [1] [2] [10] [11] [12]. For an overview of other applications, we refer to the appendix in the full version of [7]. 


\subsection{Our contribution}

We present a new algorithm for maintaining an edge orientation of a dynamic graph, with a guarantee of low out-degree and worst-case number of flips. Like many previous solutions we relate the performance to the arboricity $\alpha$ of the dynamic graph, but unlike some previous works, ours does not require knowledge of the arboricity in the general case. Our algorithm is furthermore much simpler than previous ones, and uses queues as the only under-the-hood data structure. It owes its simplicity to the fact that it greedily chooses which edge to flip.

By controlling a run-time parameter, our algorithm allows a user-specified trade-off between the out-degree and the number of flips; this was previously only possible for algorithms with amortized number of flips. Depending on the choice of the parameter, the algorithm can maintain e.g. $\mathcal{O}(\alpha+\log n)$ out-degree with $\mathcal{O}(\log n)$ flips, or $\mathcal{O}\left(\alpha \log ^{2} n\right)$ out-degree with constant flips. Various other parameter settings are possible. We match or improve all known bounds with worst-case flips, except when the arboricity is within a specific, very narrow range.

\section{Preliminaries}

The arboricity of a graph $G$ is the smallest number $t$ such that the edges of $G$ can be partitioned into $t$ forests. Several equivalent definitions are used throughout the literature. We use arboricity $(G)$ to denote the arboricity of $G$. A graph $G$ with bounded arboricity arboricity $(G) \leq \alpha$ is sparse: any induced subgraph of $G$ on $n^{\prime} \leq n$ vertices contains at most $\left(n^{\prime}-1\right) \alpha$ edges. Note that while bounded arboricity graphs have no dense neighbourhood they can still have vertices of arbitrarily high degree, e.g. stars have arboricity 1 but maximum degree $n-1$.

We say that $\mathcal{G}=G_{0}, G_{1}, G_{2}, \ldots, G_{t}$ is an edit-sequence of graphs if for each $i>0$ there exists some edge $(u, v)$ s.t. either $G_{i}=G_{i-1} \cup\{(u, v)\}$ (update $i$ is an insertion) or $G_{i}=G_{i-1} \backslash\{(u, v)\}$ (update $i$ is a deletion). We typically assume $G_{0}=\emptyset$. We say that $\mathcal{G}$ has bounded arboricity (by a number $\alpha$ ), or that $\operatorname{arboricity}(\mathcal{G}) \leq \alpha$, if $\operatorname{arboricity}\left(G_{i}\right) \leq \alpha$ for every $i$.

An orientation of a graph $G$ is a directed graph $\bar{G}$ with the same vertex and edge sets as $G$, but where an undirected edge $(u, v) \in G$ exists as the directed edge $(u, v)$ or $(v, u)$ in $\bar{G}$. We use $\operatorname{deg}(\bar{G})$ to denote the maximum out-degree of $\bar{G}$; it is a $c$-orientation if $\operatorname{deg}(\bar{G}) \leq c$. Any graph $G$ with arboricity $(G) \leq \alpha$ has an $\alpha$-orientation; to see this, partition the edges into $\alpha$ forests, pick an arbitrary root in every tree, and direct every edge towards the root of its respective tree.

We say that $\overline{\mathcal{G}}=\bar{G}_{0}, \bar{G}_{1}, \ldots, \bar{G}_{t}$ is a sequence of orientations of $\mathcal{G}$ if every $\bar{G}_{i}$ is an orientation of $G_{i}$. Similarly, $\overline{\mathcal{G}}$ is a $c$-orientation if every $\bar{G}_{i}$ is a $c$-orientation. A flip is a triple $(i, v, u)$ such that $(v, u)$ is an edge in $\bar{G}_{i-1}$ and $(u, v)$ is an edge in $\bar{G}_{i}$.

An offline c-orientation strategy $\kappa$ is some method that takes $\mathcal{G}$ and produces a $c$ orientation $\overline{\mathcal{G}}$. By abusing notation we will also use $\kappa$ to refer to the $\bar{G}$ produced by $\kappa$.

An online c-orientation algorithm $\mathcal{A}$ is analogous to the offline strategy, except that it receives $\mathcal{G}$ as a stream and has only a single $\bar{G}_{i}$ stored in memory at any time. Hence, upon receiving update $i$, it produces $\bar{G}_{i}$ as a function of $G_{i}$ and $\bar{G}_{i-1}$ and then forgets $\bar{G}_{i-1}$. We also say that $\mathcal{A}$ maintains an online $c$-orientation of $\mathcal{G}$.

We say that $\kappa$ or $\mathcal{A}$ makes $\sigma$ flips (in the worst case) if the number of flips between any two updates $i, i+1$ is at most $\sigma$, and that it makes $\sigma$ amortized flips if after any update $i$ the total amount of flips is at most $\sigma i$. 
Note the difference in wording: a strategy has access to the whole sequence $\mathcal{G}$ and produces the entire $c$-orientation at once, possibly using brute force. The online algorithm instead sees $\mathcal{G}$ as a stream of unknown length and, after every update $i$, produces only a single "current" orientation.

\section{The algorithm}

The algorithm takes an edit-sequence of graphs $\mathcal{G}$ as an online stream, and a positive integer parameter $k$. Each vertex $v$ maintains a standard FIFO queue $Q_{v}$ which holds all of its out-edges. On an insertion (deletion) update, orient the new edge arbitrarily (delete the edge via object reference) and then $k$ times pick a vertex $v$ with maximum out-degree and flip the first edge in $Q_{v}$. The book-keeping of out-degrees is trivial by using e.g. a degree-indexed array and a pointer to the maximum degree. We do not explicitly support queries. See pseudocode below for ease of reading.

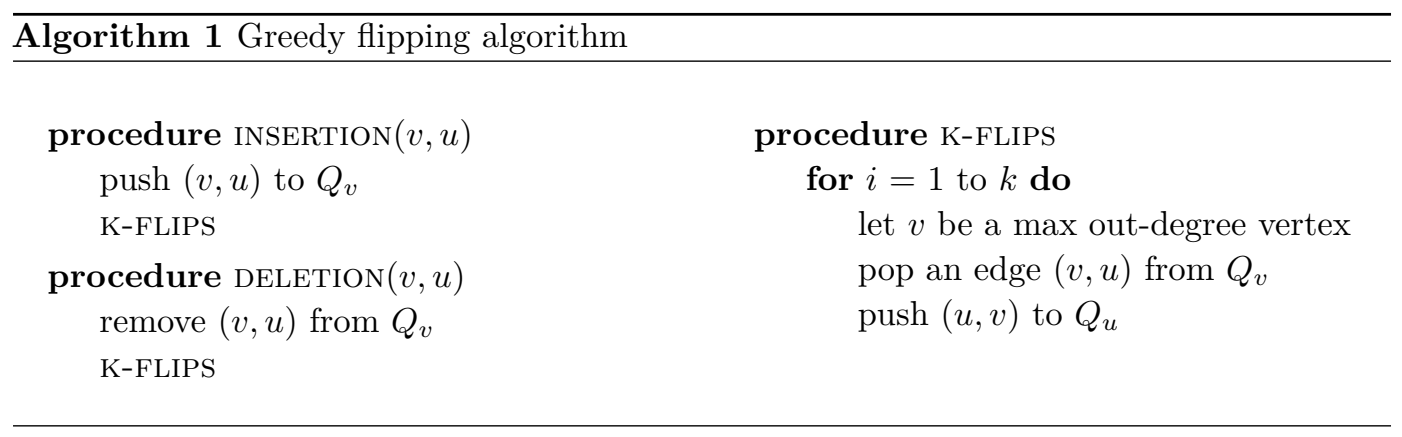

\section{Analysis}

To show the efficiency of Algorithm 1, we will prove that its out-degree is competitive to an unknown offline strategy. For given $\mathcal{G}$ and $k$, let $\delta, \sigma$ and $\varepsilon$ be values satisfying the following conditions: (i) there exists an offline $\delta$-orientation strategy $\kappa$ of $\mathcal{G}$ making at most $\sigma$ flips in the worst case, (ii) $0<\varepsilon \leq 1$, and (iii) $k \geq 1+1 / \varepsilon+2 \sigma$.

- Theorem 1. Algorithm 1 maintains an online $\mathcal{O}\left(\delta+(\delta \varepsilon+1) \log _{2} n\right)$-orientation of $\mathcal{G}$ with $k$ flips and in $\mathcal{O}(k)$ time.

Note that Algorithm 1 is completely oblivious to the values of $\delta, \sigma$ and $\varepsilon$, as well as any graph properties of $\mathcal{G}$ itself. The number of flips, and hence the running time, in Theorem 1 is trivial from the pseudocode. The rest of this section is dedicated to proving the bound on the out-degree. While the proof is quite non-trivial, the roadmap thereof is easy. We will associate potentials on all edges, such that the potential of an edge depends on where it is stored. Then we show that the total potential cannot increase, unless the maximum out-degree is $\mathcal{O}(\delta)$ in which case the potentials do not matter. Finally we re-interpret the moving of potentials as a game, where even an adversary cannot concentrate more than $\mathcal{O}((\delta \varepsilon+1) \log n)$ extra potential in any single vertex - this also (roughly) bounds the maximum out-degree.

For purposes of analysis, we consider each queue $Q_{v}$ to be two queues, the Front $F_{v}$ and $B a c k B_{v}$. Edges are always inserted into $B_{v}$, and extracted from $F_{v}$. If $F_{v}$ is empty when an edge should be extracted from $Q_{v}$, simply swap the two queues (by renaming) and then continue. It should be trivially clear that this is equivalent to using a single queue. We say an edge was flipped from $v$ and to $u$ if it was removed from $Q_{v} / F_{v}$ and inserted into $Q_{u} / B_{u}$. 
Table 2 Potential by type and placement in queue.

\begin{tabular}{|c|c|c|}
\hline & Front & Back \\
\hline Good & $1+2 \varepsilon$ & $1-\varepsilon$ \\
\hline Bad (first $3 \delta$ ) & 1 & $1+\varepsilon$ \\
\hline Bad (rest) & 1 & 1 \\
\hline
\end{tabular}

To bound the maximum out-degree, we introduce potentials on the edges. At update $i$, we say that an edge in $\bar{G}_{i}$ is good if it has the same orientation as in $\kappa\left(G_{i}\right)$ and bad otherwise. Good edges have $1+2 \varepsilon$ potential if they are in a Front queue and $1-\varepsilon$ in a Back queue. Bad edges have potential 1, except for the first $3 \delta$ bad edges in any Back queue which have potential $1+\varepsilon$. Let $p(v)$ be the sum of potentials of all edges stored in $Q_{v}, \hat{p}(\bar{G})=\max _{v} p(v)$ and $P(\bar{G})=\sum_{v} p(v)$. When we need to differentiate the potential of a vertex in a specific orientation $\bar{G}_{i}$, we use $p_{i}(v)$ to denote $p(v)$ at the time that the algorithm was storing $\bar{G}_{i}$.

Since Algorithm 1 does not know the values of $\delta$ or $\varepsilon$, it cannot determine the exact potential of a vertex. But as the following lemma shows, the out-degree of a vertex is a close approximation of its potential. We will prove the theorem by bounding the maximum potential of any vertex, which then implies a bound on its degree.

- Lemma 2. For any vertex $v, \operatorname{deg}(v)+5 \delta \varepsilon \geq p(v) \geq \operatorname{deg}(v)-\delta \varepsilon$.

Proof. For the upper bound, all edges contribute a base 1 potential, accounting for the $\operatorname{deg}(v)$ term. Note that at most $\delta$ out-edges of $v$ are good. If they are all placed in $F_{v}$, they contribute an extra $2 \delta \varepsilon$. At most $3 \delta$ bad edges in $B_{v}$ contribute an extra $\varepsilon$ each, giving at most $5 \delta \varepsilon$ extra potential in total.

For the lower bound, only good edges in $B_{v}$ can contribute less than 1 potential. Again there are at most $\delta$ of these and they contribute $\varepsilon$ less, giving at least $\operatorname{deg}(v)-\delta \varepsilon$ potential in the vertex.

Let $\beta=6 \delta \varepsilon$ be the resolution of the system. The following states that the potential of the highest-degree vertex is not too far from the maximum potential of any vertex.

- Lemma 3. Let $u$ be some vertex with maximum potential, and let $v$ be some maximum out-degree vertex. Then $p(u)-p(v) \leq \beta$.

Proof. By Lemma 2, the potential of $v$ is at least $p(v) \geq \operatorname{deg}(v)-\delta \varepsilon$ and the potential of $u$ is at most $p(u) \leq \operatorname{deg}(u)+5 \delta \varepsilon \leq \operatorname{deg}(v)+5 \delta \varepsilon$. Rearranging we get $p(u)-p(v) \leq$ $\operatorname{deg}(v)+5 \delta \varepsilon-(\operatorname{deg}(v)-\delta \varepsilon)=6 \delta \varepsilon=\beta$.

- Lemma 4. Assume a vertex $v$ has an empty $F_{v}$ and at least $4 \delta$ edges in $B_{v}$. Then swapping $F_{v}$ and $B_{v}$ does not increase $p(v)$.

Proof. The Back queue contains at most $\delta$ good edges and at least $3 \delta$ bad edges, hence exactly $3 \delta$ bad edges carry an extra $\varepsilon$ potential which is released when moving from $B_{v}$ to $F_{v}$. This $3 \delta \varepsilon$ potential is enough to raise the potential of all $\delta$ good edges from $1-\varepsilon$ to $1+2 \varepsilon$. Any surplus potential is lost.

Lemma 5. Let $v$ have out-degree at least $4 \delta$. Then flipping an edge from $v$ releases at least $\varepsilon$ potential.

Proof. By Lemma 4 we can assume $F_{v}$ is non-empty. Let $(u, v)$ be the edge moved from $F_{v}$ to $B_{u}$. Note that if the edge was previously good it is now bad, and vice versa. Hence its potential decreases either from $1+2 \varepsilon$ to at most $1+\varepsilon$, or from 1 to $1-\varepsilon$. 
- Lemma 6. Let $S$ be any suffix of the sequence of flips performed by Algorithm 1 after some update. Let $d=\operatorname{deg}(\bar{G})$ at the start of $S$. Then $\operatorname{deg}(\bar{G}) \leq d+1$ after $S$.

Proof. Note that flips can increase the maximum degree only if there are at least two vertices $u, v$ with maximum degree, and the algorithm flips an edge incident on both of them. As soon as some vertex reaches degree $d+1$, it will be the only vertex of maximum degree and immediately fall down to degree $d$ in the following flip. Consequently no sequence of flips can raise a second vertex to degree $d+1$, which is a necessary condition for raising any vertex to degree $d+2$.

- Lemma 7. Let $v$ be a vertex that had an edge flipped from it on update $i$. Then $\operatorname{deg}_{\bar{G}_{i}}(v) \geq$ $\operatorname{deg}\left(\bar{G}_{i}\right)-2$.

Proof. Take the suffix $S$ of flips that begins with the last flip from $v$. Before $S, v$ had maximum out-degree $d$. After $S, d-1 \leq \operatorname{deg}(v)$ and $\operatorname{deg}\left(\bar{G}_{i}\right) \leq d+1$ by Lemma 6 .

Consider the algorithm as it receives an update $i$. We say that the currently stored graph $\bar{G}_{i-1}$ has sufficient degree if each of the $k$ flips associated with update $i$ is from a vertex with out-degree at least $4 \delta$. Conversely, we say the graph $\bar{G}_{i-1}$ has insufficient degree if at least one of the $k$ flips is from a vertex with out-degree less than $4 \delta$.

- Lemma 8. If $\bar{G}_{i-1}$ has insufficient degree, then $\operatorname{deg}\left(\bar{G}_{i}\right)=\mathcal{O}(\delta)$.

Proof. Since some edge was flipped from a vertex with out-degree $d<4 \delta$, it follows from Lemma 6 that $\operatorname{deg}\left(\bar{G}_{i}\right) \leq d+1 \leq 4 \delta$.

- Lemma 9. If $\bar{G}_{i-1}$ has sufficient degree, then $P\left(\bar{G}_{i}\right) \leq P\left(\bar{G}_{i-1}\right)$.

Proof. Assume update $i$ is an insertion. The new edge is inserted into a Back queue, and adds at most $1+\varepsilon$ potential. The offline strategy $\kappa$ makes at most $\sigma$ flips, which causes $\sigma$ stored edges to swap their classification ("renaming") from good to bad or vice versa. A Front edge that was bad increases potential from 1 to $1+2 \varepsilon$, and a Back edge that was good increases from $1-\varepsilon$ to 1 or $1+\varepsilon$. The renaming can therefore increase the total potential by at most $2 \sigma \varepsilon$. Each flip frees $\varepsilon$ potential by Lemma 5 and the assumption of sufficient degree, so the total potential does not increase as long as $k \varepsilon \geq 1+\varepsilon+2 \sigma \varepsilon$. This is guaranteed by the choice of parameters.

If the update was instead a deletion, the flips still release $k \varepsilon$ potential while even less potential is inserted.

Note that the potential of the system can increase on both insertion and deletion updates if the graph has insufficient degree, since we cannot rely on Lemma 4 to ensure that the potential of a vertex is well-behaved when flipping edges from it. Also note that if $\kappa$ is known not to perform any flips on deletion updates, no potential gets added to the system and so our algorithm can also forgo flipping on deletions.

So far we have shown that either the maximum out-degree is $\mathcal{O}(\delta)$, or we have a nonincreasing quantity of potential and the degree of each vertex is closely approximated by its own potential. We next bound the maximum out-degree via a counter game, disassociated from the actual graph orientation, played by an adversary whose goal it is to concentrate as much potential as possible in a single counter. Counter games have been explored previously, under various names, in e.g. [4] and [9]: typically they may be thought of as two-player games where the second player is benevolent. Our game is different because the lone player is instead restricted by the concept of resolution $\beta$. 
Formally, the game is played by a single player on $n$ counters $x_{1}, \ldots, x_{n}$. Each counter $x_{i}$ will hold a non-negative real-valued weight $\left|x_{i}\right|$, and the sum of weights is a constant $\sum_{i}\left|x_{i}\right|=X$. Any such distribution of $X$ on the $n$ counters is called a game configuration $C$. Let $\hat{x}=\max _{i}\left|x_{i}\right|$ be the maximum weight at any time. The player can perform arbitrarily many iterations of the following three-step operation: (i) pick a counter $x_{i}$ and a $c>0$ such that $\left|x_{i}\right|-c \geq \max (0, \hat{x}-\beta-2)$, (ii) remove $c$ weight from $x_{i}$ and (iii) add positive weights whose sum is $c$ to any set of counters.

The player is therefore allowed to redistribute weight to arbitrary counters, but must take it in not-too-large chunks from counters that are within the resolution (here $\beta+2$ ) of the maximum counter. Before upper-bounding $\hat{x}$, we show that the player is powerful enough to simulate the movement of potentials by Algorithm 1. We say a game configuration $C$ dominates a graph orientation $\bar{G}$ if $\left|x_{j}\right| \geq p\left(v_{j}\right)$ for every $j$.

- Lemma 10. Let $i$ be an update such that $\bar{G}_{i-1}$ has sufficient degree. Let $C$ be a game configuration that dominates $\bar{G}_{i-1}$. Then the player can reach a game configuration $C^{\prime}$ that dominates $\bar{G}_{i}$.

Proof. We need to show that if some vertex gains potential (so its corresponding counter no longer dominates it), then we can safely take enough weight from other counters to fill that 'gap'. Keep in mind that the total potential does not increase (Lemma 9). Since the player is allowed to redistribute weight to any counter, we let the gaps be filled in arbitrary order and only show that enough weight can be taken from other counters to make up the difference. If $\hat{x}>\hat{p}\left(\bar{G}_{i-1}\right)$ then greedily take weight from all counters greater than $\hat{p}\left(\bar{G}_{i-1}\right)$ to get $\hat{x}=\hat{p}\left(\bar{G}_{i-1}\right)$.

Let $v_{j}$ be a vertex that had an edge flipped from it. Then its resulting out-degree is $\operatorname{deg}_{\bar{G}_{i}}\left(v_{j}\right) \geq \operatorname{deg}\left(\bar{G}_{i}\right)-2$ (Lemma 7$)$ and its potential is $p_{i}\left(v_{j}\right) \geq \operatorname{deg}_{\bar{G}_{i}}\left(v_{j}\right)-\delta \varepsilon \geq$ $\operatorname{deg}\left(\bar{G}_{i}\right)-2-\delta \varepsilon$ (Lemma 2$)$. Also by Lemma 2 the maximum potential in the system is $\hat{p}\left(\bar{G}_{i}\right) \leq \operatorname{deg}\left(\bar{G}_{i}\right)+5 \delta \varepsilon$. Hence the final potential of $v_{j}$ is within $6 \delta \varepsilon+2=\beta+2$ of the maximum potential. As the rules of the counter game allow us to take weight up to $\beta+2$ from the maximum counter, then however much potential $v_{j}$ lost we can take at least the same amount of weight from its corresponding counter $x_{j}$.

Conversely, if a vertex loses potential but its resulting potential is not at least $\hat{p}\left(\bar{G}_{i}\right)-\beta-2$, it must have lost that potential due to deletion or renaming rather than flipping. Its counter can safely be left untouched and still dominate the potential of the vertex.

Since the sum of potential decreases (by flipping) is at least as large as the sum of increases (for any reason) (Lemma 9), and for any vertex that lost potential by flipping we can remove at least as much weight from its counter, then we can redistribute enough weight to raise the too-low counters to again dominate their respective vertex potentials. The updated counters form a game configuration that dominates $\bar{G}_{i}$.

- Lemma 11. Let $\bar{G}_{a}, \ldots, \bar{G}_{b}$ be any sequence of orientations such that $\bar{G}_{i}$ has sufficient degree for every $a \leq i \leq b$. Consider a game with starting configuration $C_{a}$ that dominates $\bar{G}_{a}$, with $\hat{x}=\hat{p}\left(\bar{G}_{a}\right)$. Then the player can reach game configurations $C_{a}, \ldots, C_{b}$ where $C_{i}$ dominates $\bar{G}_{i}$ for every $a \leq i \leq b$.

Proof. For every $a<i \leq b$ iterate Lemma 10 on $C_{i-1}$ to create $C_{i}$.

We now let an adversary play the game, with the goal to increase $\hat{x}$ as much as possible. For simplicity we assume that every counter is raised to $\hat{x}$ as the starting configuration. For $j=-1,0,1,2, \ldots$ let $\ell_{j}=X / n+j(\beta+2)$ be weight level $j$. A counter $x_{i}$ is above 
level $j$, or above $\ell_{j}$, if $\left|x_{i}\right| \geq \ell_{j}$. Let $X_{j}=\sum_{i=1}^{n} \max \left(0,\left|x_{i}\right|-\ell_{j}\right)$ be the weight above $\ell_{j}$, and $\bar{X}_{j}=X-X_{j}$ the weight below $\ell_{j}$. We say a counter $x_{i}$ contributes $\max \left(0,\left|x_{i}\right|-\ell_{j}\right)$ to $X_{j}$ and $\min \left(\left|x_{i}\right|, \ell_{j}\right)$ to $\bar{X}_{j}$.

- Lemma 12. Let $j$ be a weight level such that $\ell_{j} \leq \hat{x}$. Let the player make any sequence of moves that maintain the condition $\ell_{j} \leq \hat{x}$. Then $X_{j-1}$ does not increase.

Proof. Note that any counter $x_{i}$ contributes $\min \left(\left|x_{i}\right|, \ell_{j-1}\right)$ to $\bar{X}_{j-1}$. By assumption there will always be a counter $x_{k}$ with $\ell_{j} \leq\left|x_{k}\right|$. Hence the resolution rule prevents the player from making any counter contribute less to $\bar{X}_{j-1}$ than it already does. Since $X$ is a constant and $\bar{X}_{j-1}$ is non-decreasing, $X_{j-1}=X-\bar{X}_{j-1}$ is non-increasing.

Since $\ell_{0}=X / n$ is the average weight of all counters, it must always be the case that $\hat{x} \geq \ell_{0}$ and $X_{-1} \leq n(\beta+2)$.

- Lemma 13. Let $j$ be a weight level such that $\ell_{j} \leq \hat{x} \leq \ell_{j+1}$. Let the player make any sequence of moves that maintain the condition $\ell_{j} \leq \hat{x} \leq \ell_{j+1}$. Then $2 X_{j} \leq X_{j-1}$.

Proof. By Lemma $12, X_{j-1}$ is a non-increasing amount. Let $x_{i}$ be any counter that will contribute some positive weight $w$ to $X_{j}$. Since the player maintains that $\hat{x} \leq \ell_{j+1}$, no counter will be able to contribute more than $\ell_{j+1}-\ell_{j}=\beta+2$ to $X_{j}$, i.e. $0<w \leq \beta+2$. Then $x_{i}$ must contribute $w+\beta+2$ to $X_{j-1}$. Hence any counter that contributes to $X_{j}$ contributes at least twice as much to $X_{j-1}$, and $2 X_{j} \leq X_{j-1}$.

The player is therefore stuck in the following dilemma: once $\hat{x}$ reaches some level $\ell_{j}$, only a bounded amount $X_{j-1}$ of weight remains available to redistribute. But once $\hat{x}$ reaches $\ell_{j+1}$, only the weight above $\ell_{j}$ will be possible to redistribute. Therefore, in order to concentrate as much weight as possible above $\ell_{j+2}$, the player must first maximize $X_{j}$ without any counter actually reaching above $\ell_{j+1}$.

- Lemma 14. The player cannot increase $\hat{x}$ to $\ell_{1+\log _{2} n}$.

Proof. Assume $\hat{x} \geq \ell_{\log _{2} n}$. By alternatingly iterating Lemma 12 and Lemma 13, the weight above $\ell_{\log _{2} n}$ is $X_{\log _{2} n} \leq\left(\frac{1}{2}\right)^{1+\log _{2} n} X_{-1}=\frac{1}{2 n} X_{-1} \leq \frac{1}{2 n} n(\beta+2)<\beta+2$. Since the weight is strictly less than $\beta+2$, even concentrating all of it in a single counter is not enough to make that counter reach $\ell_{1+\log _{2} n}$. Hence $\hat{x}<\ell_{1+\log _{2} n}$.

We are now ready to prove the out-degree part of Theorem 1.

Proof of Theorem 1. Either the graph orientation has insufficient degree and maximum outdegree $\mathcal{O}(\delta)$ (Lemma 8) or it has non-increasing potential (Lemma 9) which is dominated by a counter game where the starting weight of any counter is $\mathcal{O}(\delta)$ (Lemma 11). By Lemma 14, the maximum counter is $\hat{x}<\ell_{1+\log _{2} n}=\mathcal{O}(\delta)+\left(1+\log _{2} n\right)(\beta+2)$. By Lemma $2, \operatorname{deg}(v) \leq p(v)+\delta \varepsilon$, and therefore any vertex has out-degree bounded by $\mathcal{O}(\delta)+\left(1+\log _{2} n\right)(\beta+2)+\delta \varepsilon=$ $\mathcal{O}\left(\delta+(\delta \varepsilon+1) \log _{2} n\right)$.

\section{De-amortizing offline strategies}

In the previous work by Brodal and Fagerberg [3], their amortized algorithm is shown competitive with an offline strategy with bounded amortized number of flips, and hence subsequently published strategies have focused on achieving good amortized bounds. However, for our algorithm analysis, we require an offline strategy with worst-case flips per update. 
In this section we show one way to de-amortize offline strategies. Our technique does not generalize to every offline strategy, but relies on the special structure inherent to the strategies of both [7] and [5]. These strategies partition the edit-sequence into blocks of consecutive updates, with some length $\lambda$. No flips occur within a block, only in the seams between two blocks. The amortized flip complexity of these strategies is therefore simply the maximum number of flips between two blocks, divided by the length $\lambda$ of the preceding block.

Since no flips are allowed within a block, the strategy is required to find an orientation of the union of all graphs $G_{i}, \ldots, G_{i+\lambda-1}$ within a block. The maximum out-degree of the entire strategy is therefore upper bounded by the maximum out-degree of any oriented union-graph. Higher $\lambda$ gives a less sparse union-graph, necessitating higher out-degree, but also allows for a better amortized flip complexity. The following theorem shows a simple way of de-amortizing strategies with this structure, by taking all the flips between two blocks and spreading them evenly over the updates in the later block.

- Theorem 15. Let $\kappa$ be a $\delta$-orientation strategy of $\mathcal{G}$ where, for arbitrary $\lambda$, any update with $\sigma \lambda$ flips is followed by at least $\lambda-1$ updates with no flips. Then there exists a $2 \delta$-orientation strategy of $\mathcal{G}$ making $\sigma$ flips in the worst case.

Note that if the last block of flips is not followed by $\lambda-1$ updates due to $\mathcal{G}$ ending, then one can pad $\mathcal{G}$ to appropriate length by repeatedly inserting and removing a dummy edge after the end of $\mathcal{G}$. Also note that $\lambda$ can vary within the same sequence - blocks do not need to be of uniform length.

Proof. Let $i$ be an update where $\kappa$ performs a set of $\lambda \sigma$ flips. Let $F$ be the set of flipped edges. Let $\kappa^{\prime}$ be an offline strategy with the same edge orientations as $\kappa$ except on updates $i, \ldots, i+\lambda-1$. On any insertion update $i, \ldots, i+\lambda-1$, let $\kappa^{\prime}$ orient the new edge in the same direction as $\kappa$. Furthermore, on each update $i, \ldots, i+\lambda-1, \kappa^{\prime}$ takes $\sigma$ arbitrary edges in $F$, removes them from $F$, and flips them.

Then $F$ will be empty after update $i+\lambda-1$, so $\kappa\left(G_{i+\lambda-1}\right)=\kappa^{\prime}\left(G_{i+\lambda-1}\right)$. At all times $F$ forms a $\delta$-orientation, since $F$ is a subset of $\kappa\left(G_{i-1}\right)$. Similarly, $\kappa^{\prime}\left(G_{j}\right) \backslash F$ is $\delta$-orientation for every $i \leq j \leq i+\lambda-1$, since they are a subset of $\kappa\left(G_{j}\right)$. Hence $\kappa^{\prime}$ is a $2 \delta$-orientation. Finally, $\kappa^{\prime}$ performs at most $\sigma$ flips per update between updates $i$ and $i+\lambda-1$; exhaustively perform the same transformation on all of $\kappa$ for $\sigma$ flips on any update.

\section{Discussion}

With our two theorems proven, we can relate the algorithm to known offline strategies and achieve the following corollaries. In all of the following, $\mathcal{G}$ is an arbitrary edit-sequence with $\operatorname{arboricity}(\mathcal{G}) \leq \alpha$.

Kowalik [8] presents an offline $\mathcal{O}(\alpha \log n)$-orientation strategy making 1 amortized flip. Using Theorem 15 we can de-amortize it to an offline $\mathcal{O}(\alpha \log n)$-orientation strategy making 1 flip in the worst case, giving the following two corollaries.

Corollary 16. For a positive function $f(n)=\mathcal{O}(\log n)$, Algorithm 1 maintains an $\mathcal{O}\left(\frac{\alpha \log ^{2} n}{f(n)}\right)$-orientation with $k=3+\lceil f(n)\rceil$ flips.

Proof. Let $\delta=\mathcal{O}(\alpha \log n), \sigma=1$ and $\varepsilon=1 / f(n)$. Then the algorithm maintains out-degree $\mathcal{O}\left(\alpha \log n+\left(\frac{\alpha \log n}{f(n)}+1\right) \log n\right)=\mathcal{O}\left(\frac{\alpha \log ^{2} n}{f(n)}\right)$.

- Corollary 17. Algorithm 1 maintains an $\mathcal{O}\left(\alpha \log ^{2} n\right)$-orientation of $\mathcal{G}$ with $k=4$ flips. 
Proof. Let $f(n) \equiv 1$ in Corollary 16 .

Corollary 17 is the first result with $\mathcal{O}(1)$ worst-case flips. Compared to [8] (with $\mathcal{O}(1)$ amortized flips), it incurs an extra $\mathcal{O}(\log n)$ factor on the out-degree, but avoids the $\Omega(n)$ worst-case flips which that algorithm can experience.

Brodal and Fagerberg [3] give an offline $\mathcal{O}(\alpha)$-orientation strategy with $\mathcal{O}(\log n)$ flips in the worst case. It only makes flips on insertion updates.

- Corollary 18. Algorithm 1 maintains an $\mathcal{O}(\alpha+\log n)$-orientation of $\mathcal{G}$ with $k=\mathcal{O}(\log n)$ flips.

Proof. Let $\delta=\mathcal{O}(\alpha), \sigma=\mathcal{O}(\log n)$ and $\varepsilon=1 / \log n$. Then the algorithm maintains out-degree $\mathcal{O}\left(\alpha+\left(\frac{\alpha}{\log n}+1\right) \log n\right)=\mathcal{O}(\alpha+\log n)$.

He et al. [5] give an offline $\mathcal{O}(\alpha \sqrt{\log n})$-orientation strategy making $\mathcal{O}(\sqrt{\log n})$ amortized flips, which we de-amortize using Theorem 15.

- Corollary 19. Algorithm 1 maintains an $\mathcal{O}(\alpha \log n)$-orientation of $\mathcal{G}$ with $k=\Theta(\sqrt{\log n})$ flips.

Proof. Let $\delta=\mathcal{O}(\alpha \sqrt{\log n}), \sigma=\mathcal{O}(\sqrt{\log n})$ and $\varepsilon=1 / \sqrt{\log n}$. Then the algorithm maintains out-degree $\mathcal{O}\left(\alpha \sqrt{\log n}+\left(\frac{\alpha \sqrt{\log n}}{\sqrt{\log n}}+1\right) \log n\right)=\mathcal{O}(\alpha \log n)$.

- Corollary 20. Algorithm 1 maintains an $\mathcal{O}(\log n)$-orientation of $\mathcal{G}$ with $k=\mathcal{O}(\alpha \sqrt{\log n})$ flips, if $\alpha=\mathcal{O}(\sqrt{\log n})$.

Proof. Let $\delta=\mathcal{O}(\alpha \sqrt{\log n}), \sigma=\mathcal{O}(\sqrt{\log n})$ and $\varepsilon=1 / \alpha \sqrt{\log n}$. Then the algorithm maintains out-degree $\mathcal{O}\left(\alpha \sqrt{\log n}+\left(\frac{\alpha \sqrt{\log n}}{\alpha \sqrt{\log n}}+1\right) \log n\right)=\mathcal{O}(\log n)$.

Corollary 19 is an improvement over [7] in the flip complexity for edit-sequences with arboricity bounded by a constant. For $\alpha=\mathcal{O}(\sqrt{\log n} / \log \log n)$, Corollary 20 matches or improves the flip complexity from [7], albeit with a slightly worse degree bound, and only if $\alpha$ is known. If $\alpha$ is both $\mathcal{O}(\sqrt{\log n})$ and $\omega(\sqrt{\log (n)} / \log \log n)$ we are narrowly outperformed by [7], by no more than an $\mathcal{O}(\log \log n)$ factor.

Corollary 19 also nearly matches the degree bound in [5] but with worst-case flips instead of amortized. Corollary 18 matches the bounds in [7] for general arboricity and improves on their flip complexity if $\alpha=\omega(\log n)$. Furthermore, if $\alpha=\Omega(\log n)$, Corollary 18 matches the amortized bounds from [3].

\subsection{Reverse trade-off}

Compared to an offline strategy, our analysis lends itself to a trade-off in one direction, getting (at most) an $\mathcal{O}(\log n)$ factor on the out-degree for a constant factor on the number of flips. It allows us to perform much fewer flips than in [7] at the price of weaker degree bounds. A trade-off in the opposite direction would also be highly desirable, achieving out-degree (closer to) $\mathcal{O}(\delta)$ by making $\Omega(\sigma)$ flips. We have only found a very weak such trade-off:

- Lemma 21. Algorithm 1 can maintain an $\mathcal{O}(\alpha)$-orientation of $\mathcal{G}$ with $k=\mathcal{O}(\alpha n)$ flips. 
Proof. Let $\delta=\mathcal{O}(\alpha)$ and $\varepsilon=1$ (the value of $\sigma$ is inconsequential). Then each edge holds between 0 and 3 potential. And since any $G_{i}$ has at most $\alpha n$ edges (by definition of arboricity), the total potential is between 0 and $3 \alpha n$. Furthermore each flip releases 1 potential from the system, contingent on the graph having sufficient degree (Lemma 5). Hence after performing at most $3 \alpha n$ flips on any starting orientation $\bar{G}_{i}$, we must reach a state where the next flip does not release potential, contradicting Lemma 5, and so by Lemma 8 the graph has out-degree at most $4 \delta=\mathcal{O}(\alpha)$ after all flips.

Lemma 21 only matches the worst-case bound of the algorithm in [3], which has drastically better amortized performance. Hence it should not be used in practice. Still, we believe a stronger reverse trade-off is possible and conjecture the following:

- Conjecture 22. For some function $f$, Algorithm 1 maintains an online $\mathcal{O}\left(\delta+\frac{\sigma+1}{f(k)} \delta \log n\right)$ orientation of $\mathcal{G}$ with $k$ flips and in $\mathcal{O}(k)$ time.

\subsection{Dynamic arboricity}

Throughout the paper we have done all our performance analysis against a static arboricity bound, i.e. a bound on the greatest arboricity seen anywhere in the edit-sequence. An interesting issue arises if the sequence contains contiguous sub-sequences, of non-trivial length, with higher or lower arboricity than elsewhere in the sequence. Some previous algorithms, e.g. one of the algorithms in [7] and the non-amortized algorithm in [5], adapt to increasing and decreasing arboricity automatically.

Our analysis immediately adapts to sequences with increasing arboricity, since the analysis can be performed on any prefix (or contiguous sub-sequence) of $\mathcal{G}$. In the case of periods with lower arboricity than earlier in the sequence, our algorithm obeys the new arboricity if the maximum out-degree is already within that new bound. In other words, if the maximum out-degree is already bounded relative to the new arboricity, then it will remain so. However, if the arboricity falls enough that the current maximum out-degree breaks the new bounds, our analysis does not require the maximum out-degree to decrease accordingly. Intuitively, using a $k$ strictly larger than $1+1 / \varepsilon+2 \sigma$ (thus experiencing a net loss of total potential with every update) should force the maximum out-degree to tend towards the updated degree bounds, similar to the proof of Lemma 21. However, we do not have a formal argument for this.

\subsection{Open problems}

For all known strategies that maintain out-degree $\delta$ with $\sigma$ (amortized) flips, it holds that $\delta \sigma=\Omega(\alpha \log n)$ and most achieve $\delta \sigma=\Theta(\alpha \log n)$. Can one design a strategy with $\delta \sigma=o(\alpha \log n) ?$

\section{References}

1 Aaron Bernstein and Cliff Stein. Fully dynamic matching in bipartite graphs. arXiv preprint arXiv:1506.07076, 2015.

2 Aaron Bernstein and Cliff Stein. Faster fully dynamic matchings with small approximation ratios. In Proceedings of the Twenty-Seventh Annual ACM-SIAM Symposium on Discrete Algorithms, pages 692-711. Society for Industrial and Applied Mathematics, 2016.

3 Gerth Stølting Brodal and Rolf Fagerberg. Dynamic representation of sparse graphs. In Proceedings 6th International Workshop on Algorithms and Data Structures (WADS), volume 1663 of Lecture Notes in Computer Science, pages 342-351. Springer, 1999. 
4 Paul Dietz and Daniel Sleator. Two algorithms for maintaining order in a list. In Proceedings 19th Annual ACM Symposium on Theory of Computing (STOC), pages 365-372. ACM, 1987.

5 Meng He, Ganggui Tang, and Norbert Zeh. Orienting dynamic graphs, with applications to maximal matchings and adjacency queries. In Proceedings 25th International Symposium on Algorithms and Computation (ISAAC), volume 8889 of Lecture Notes in Computer Science, pages 128-140. Springer, 2014.

6 Sampath Kannan, Moni Naor, and Steven Rudich. Implicit representation of graphs. SIAM Journal on Discrete Mathematics, 5(4):596-603, 1992.

7 Tsvi Kopelowitz, Robert Krauthgamer, Ely Porat, and Shay Solomon. Orienting fully dynamic graphs with worst-case time bounds. In Proceedings 41st International Colloquium Automata, Languages, and Programming (ICALP), Part II, volume 8573 of Lecture Notes in Computer Science, pages 532-543. Springer, 2014.

8 Łukasz Kowalik. Adjacency queries in dynamic sparse graphs. Information Processing Letters, 102(5):191-195, 2007.

9 Christos Levcopoulos and Mark H. Overmars. A balanced search tree with $O(1)$ worst-case update time. Acta Informatica, 26(3):269-277, 1988.

10 Ofer Neiman and Shay Solomon. Simple deterministic algorithms for fully dynamic maximal matching. ACM Transactions on Algorithms (TALG), 12(1):7, 2016.

11 David Peleg and Shay Solomon. Dynamic $(1+\varepsilon)$-approximate matchings: a densitysensitive approach. In Proceedings of the Twenty-Seventh Annual ACM-SIAM Symposium on Discrete Algorithms, pages 712-729. Society for Industrial and Applied Mathematics, 2016.

12 Shay Solomon. Fully dynamic maximal matching in constant update time. In Foundations of Computer Science (FOCS), 2016 IEEE 57th Annual Symposium on, pages 325-334. IEEE, 2016. 